\title{
Infliximab in the treatment of Budd-Chiari syndrome in Behçet's disease
}

\author{
L.R. Santos, J. Rigueira, A.P. Vilas \\ Service of Internal Medicine 2B, Hospital of Santa Maria/Centro Hospitalar Lisboa Norte, Portugal
}

\begin{abstract}
SUMMARY
Behçet's disease is a chronic autoinflammatory disorder that usually presents with recurrent oral and genital ulcers, uveitis, skin lesions and arthritis. Any organ, however, can be involved and in a subgroup of patients the large vascular lesions are the predominant feature (vasculo-Behçet disease). We report a case of a 27-yearold man with Behçet's disease who developed Budd-Chiari syndrome while on immunosuppressive therapy. Infliximab resulted in a rapid and total clinical remission. Literature data have shown that antitumor necrosis factor alpha (anti-TNF- $\alpha$ ) agents including infliximab can suppress disease activity but there is no long-term experience on tapering and/or discontinuation of treatment under clinical remission. Our case supports the use of infliximab in vasculo-Behçet patients.
\end{abstract}

Key words: Behçet's disease; venous thrombosis; Budd-Chiari; infliximab.

\section{INTRODUCTION}

B ehçet's disease (BD) is a chronic multisystem autoinflammatory disease of unknown etiopathogenesis that develops in the third decade of life and has a relapsing and remitting course (1). Young men are at higher risk of more severe disease, particularly with vascular involvement. According to the International Criteria (ICBD), BD is diagnosed if a patient scores 4 or more points (ocular lesions, genital and oral ulcers - 2 points; skin lesions, neurological manifestations, vascular manifestations, positive pathergy test - 1 point) (2). Vascular involvement occurs in $6.3-51.6 \%$ of patients (3). Deep venous thrombosis (DVT) is the most frequent vascular event and it is also the first vascular manifestation in most cases (3). Thrombosis of hepatic veins or suprahepatic inferior vena cava (Budd-Chiari syndrome - BCS) is a rare and potentially lethal form of Behçet's disease (4). Recent reports reveal inhibition of tumor necrosis factor alpha (TNF- $\alpha$ ) has dramatic efficacy for the successful treatment of various types of $\mathrm{BD}$, including vasculoBD. However, it is completely unknown whether we can discontinue anti-TNF- $\alpha$ agents in patients with severe vasculo-BD under clinical remission. The authors report a case of a BD-BCS that was successfully treated with infliximab.

\section{CASE REPORT}

A 27-year-old man was referred to our Autoimmune Unit because of recurrent episodes of DVT of the lower limbs and erythema nodosum (EN). As a child, he had recurrent tonsillitis and at the age of 23 years, he had his first DVT (femoro-popliteal). Hypercoagulable states and autoimmune diseases were excluded. He began warfarin but despite adequate anticoagulation he had several more DVT of the lower limbs. At the age of 25 he had his first episode of EN and at 27 his second. Further detailed medical history showed that he had had recurrent oral ulcers. The pathergy test was positive. He scored 5 points in ICBD and therefore was diagnosed with BD. He began corticosteroids but EN persisted and colchicine and then azathioprine were added. Despite treatment, at the age of 28 years, he developed a right iliofemo-
Corresponding author: Liliana R. Santos Service of Internal Medicine 2B, Hospital of Santa Maria/ Centro Hospitalar Lisboa Norte, Portugal E-mail: santos.lilianaribeiro@gmail.com 

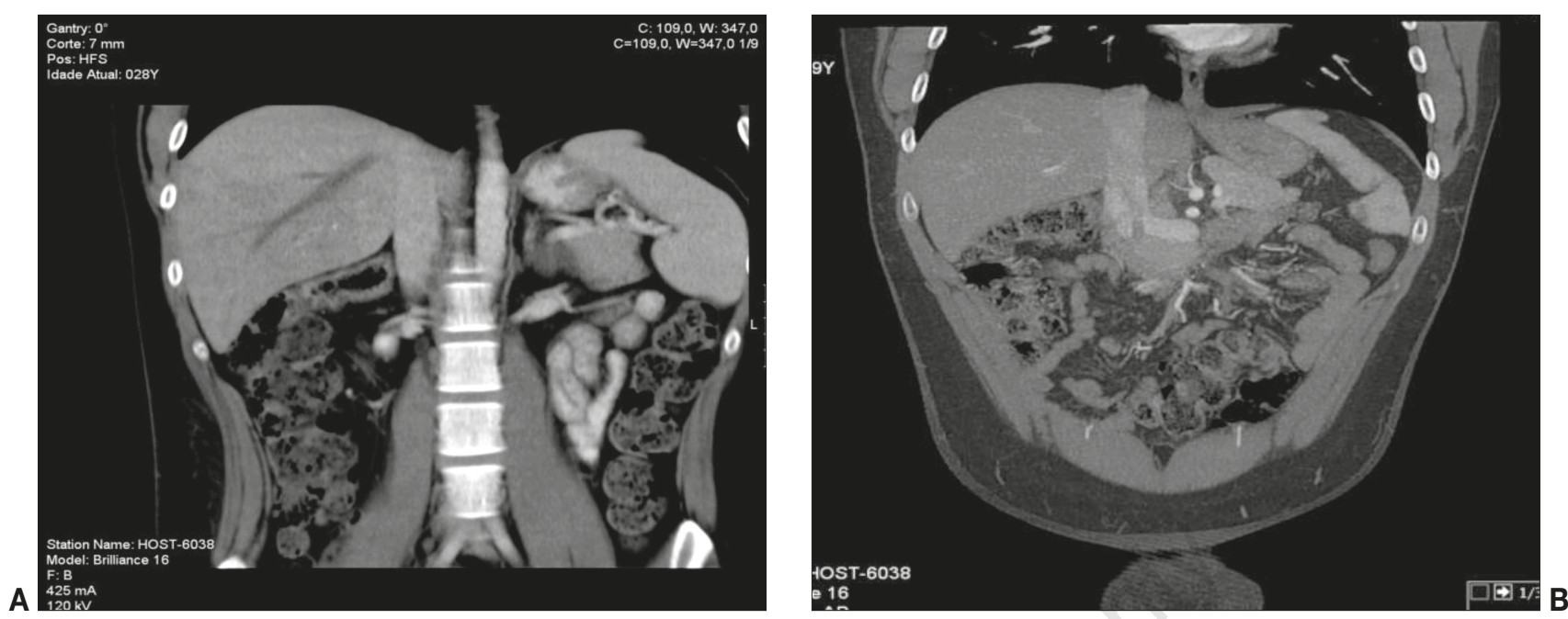

Figure 1 - A) Suprahepatic vein thrombosis; B) after infliximab treatment the venous thrombosis is no longer visible.

ral DVT and an extensive thrombosis of right suprahepatic vein (Figure 1A). He began monthly infliximab $(5 \mathrm{mg} / \mathrm{kg})$ and rapid clinical improvement occurred. Three months later the CT scan showed no evidence of thrombi in the hepatic veins (Figure 1B). Over the next months, corticosteroids, colchicine and azathioprine were discontinued. After 12 months of infliximab, the treatment was extended to once every 8 weeks. Anticoagulation was switched to apixaban and 6 months later it was stopped. The patient remains disease-free more than 3 years after commencement of infliximab and more than 2 years after anticoagulation cessation.

\section{DISCUSSION AND CONCLUSIONS}

TNF- $\alpha$ targeted treatment in vasculo-BD may be effective. TNF- $\alpha$ is considered to play a crucial role in the BD inflammatory pathway. Its expression is increased in the peripheral blood of patients with active BD and TNF- $\alpha$ mRNA expression decreased following TNF- $\alpha$ blocker treatment. We present a severe case of BCS, which is known to represent less than $3 \%$ of the vasculo-Behçet Disease. In our case, the suprahepatic vein thrombosis occurred under treatment with corticosteroids and azathioprine. Infliximab was initiated with a total remission documented at the third month of treatment. After 1 year, infliximab was reduced to once every 8 weeks. Response was maintained even after corticosteroid, azathioprine and anticoagulation discontinuation. Until now, infliximab has been well tolerated and no side effects occurred. We speculate that earlier initiation of treatment with infliximab may lead to a better outcome. It is known that treatment choices for BD-BCS are not standardized due to the lack of high quality randomized controlled trials. Experience exists with infliximab in refractory ocular involvement in BD and a high efficacy rate has been documented (5). Infliximab has also been used as an off-label agent in vasculo-Behçet Disease, with promising results (5) - so much so that in August of 2015, infliximab became covered by the Japanese National Health Insurance in refractory cases of specific types of BD including vasculo-Behçet Disease (6). EULAR guidelines 2018 suggested that a long-term remission after withdrawal of successful anti-TNF treatment combined with azathioprine given for 2 years is feasible in a good proportion of patients with sight-threatening ocular disease (7).

Although in rheumatic disorders, tapering and discontinuation of biologics are feasible, it is still unknown whether biologic treatments can be discontinued in major ves- 
sels thrombosis after achievement of clinical remission. To our knowledge, no data exist to support infliximab discontinuation in our patient. Some authors have stopped treatment after one year of sustained remission with no relapses during at least 13 months of follow up, but these patients had vascular involvement of non-life-threatening major vessels and immunosuppressive therapy was never stopped (6).

Our case not only reinforces the idea that anticoagulation is not indicated in vasculoBehçet Disease patients, but also supports the utilization of Infliximab in vasculoBehçet Disease and reinforces that careful judgement is necessary when deciding discontinuation of a biologic treatment in severe conditions. Therefore, further investigation and large cohort studies are necessary to establish clear criteria for biologic treatment discontinuation in severe vasculo-Behçet Disease.

\section{Learning points}

- Diagnosing BD may be difficult since manifestations are nonspecific and usually not simultaneous. Vascular involvement with venous inflammation leads to adherent thrombus formation (without thromboembolism) and arterial inflammation facilitates aneurysm formation.

- DVT is the most frequent vascular event and it is also the first vascular manifestation in most cases. DVT should be treated with immunosuppressive agents such as corticosteroids, azathioprine, cyclophosphamide or cyclosporine A.

- In BCS, monthly pulse cyclophosphamide is recommended. However, mortality rates in the first months remain around $77 \%$.

- Infliximab, an anti-TNF $\alpha$ agent, inhibits the activation of infiltrated mono- nuclear cells and therefore diminishes vascular inflammation and seems to be a good alternative in BCS-BD.

- Current data does not orientate on infliximab duration and discontinuation of treatment in BCS-BD. Our case not only supports the use of infliximab in vasculo-Behçet Disease but also reinforces the idea that anticoagulation is not indicated in vasculo-Behçet Disease patients.

Conflict of interests: The authors declare that there is no conflict of interests regarding the publication of this paper.

\section{REFERENCES}

1. Karincaoglu Y, Borlu M, Toker SC, et al. Demographic and clinical properties of juvenileonset Behçet's disease: A controlled multicenter study. J Am Acad Dermatol. 2008; 58: 579-84.

2. Davatchi F. International Team for the Revision of the International Criteria for Behcet's Disease. 2013 The International Criteria for Behcet's Disease (ICBD): uno studio collaborativo di 27 paesi sulla sensibilità e la specificità dei nuovi criteri. J Eur Acad Dermatol Venereol. 2014; 28: 338-47.

3. Tascilar K, Melikoglu M, Ugurlu S, et al. Vascular involvement in Behçet's syndrome: a retrospective analysis of associations and the time course. Rheumatology. 2014; 53: 2018-21.

4. Desbois AC, Rautou PE, Biard L, et al. Behçet's disease in Budd-chiari syndrome. Orphan J Rare Dis. 2014; 9: 104.

5. Saleh Z, Arayssi T. Update on the therapy of Behçet disease. Ther Adv Chronic Dis. 2014; 5: 112-34.

6. Nakamura A, Miyamura T, Suematsu E. Successful discontinuation of Infliximab in a refractory case of Vasculo-Behçet's disease. Case Rep Rheumatol. 2016; 2016: 1467583.

7. Hatemi G, Christensen R, Bang D, et al. Aggiornamento 2018 delle raccomandazioni EULAR per la gestione della sindrome di Behçet. Ann Rheum Dis. 2018; 0: 1-11. 